EPJ Web of Conferences 59, 13008 (2013)

DOI: $10.1051 /$ epjconf/20135913008

(C) Owned by the authors, published by EDP Sciences, 2013

\title{
Absolute $\mathrm{K} \alpha$ line spectroscopy for cone-guided fast-ignition targets
}

H. Nishimura ${ }^{1, a}$, Z. Zhang ${ }^{1}$, T. Namimoto ${ }^{1}$, S. Fujioka ${ }^{1}$, M. Koga ${ }^{1}$, H. Shiraga ${ }^{1}$, T. Ozaki ${ }^{2}$, T. Iwawaki ${ }^{3}$, T. Morioka ${ }^{3}$, K. Morita ${ }^{3}$, H. Habara ${ }^{1,3}$, K.A. Tanaka ${ }^{3}$, M. Nishikino ${ }^{4}$, T. Kawachi' ${ }^{4}$ A. Sagisaka ${ }^{4}$, S. Orimo ${ }^{4}$, A.S. Pirozhkov ${ }^{4}$, K. Ogura ${ }^{4}$, A. Yogo ${ }^{4}$, H. Kiriyama ${ }^{4}$, K. Kondo ${ }^{4}$, T. Shimomura ${ }^{4}$, S. Kanazawa ${ }^{4}$, Y. Okano ${ }^{5}$, FG-02 Experimental Campaign Team ${ }^{1}$ and H. Azechi ${ }^{1}$

1 Institute of Laser Engineering, Osaka U., Suita, Osaka 565-0871, Japan

2 Institute for Fusion Science, Toki, Gifu 509-5292, Japan

3 Graduate School of Engineering, Osaka U., Suita, Osaka 565-0871, Japan

4 Kansai Photon Science Institute, JAEA, Kyoto 619-0215, Japan

5 Institute for Molecular Science, Okazaki, Aichi 444-8585, Japan

\begin{abstract}
Absolute $\mathrm{K} \alpha$ line spectroscopy is proposed to study laser-plasma interactions taking place in cone-guided fast ignition targets. A transmission-type spectrometer was developed, and its absolute sensitivity was measured with laser-generated $\mathrm{x}$-rays and radioisotopes, showing good agreement with theoretical predictions. The absolute yield of $\mathrm{Au} \mathrm{K} \alpha$ line was mesured in the fast ignition experimental campaign perfomed at ILE, Osaka University. Assuming a single Maxwellian electron spectrum and oneway travel of hot electrons through the cone, energy transfer efficiency of incident LFEX laser to hot electrons was derived.
\end{abstract}

\section{INTRODUCTION}

Fast ignition is recognized as a promising pathway to efficient thermonuclear fusion in laser-driven inertial confinement fusion. A cone-guided CD-shell has been used as a base-line target for the fast ignition experiment. It has long been expected to provide more quantitative information about the hot electron generation and transport in the cone than those derived only with $\mathrm{x}$-ray imaging and neutron detection. Hot electron spectra observed with an electron spectrometer suffer from substantial modification: Although the major fraction of hot electron energy is carried by the component around 1-2 MeV, corresponding spectral information is missing due to the self-generated plasma potential. To solve this problem, Bremsstrahlung x-ray spectra, as a consequence of hot electron propagation in dense plasma, are measured [1]. In addition to this, we propose an absolute $\mathrm{K} \alpha$ line spectroscopy dedicated to quantitative measurement of hot electron generation and transport in the high- $Z$ target. This method has the advantage over the Bremsstrahlung measurement that it provides local information about the hot electrons propagating through specific materials composing the cone-guided target. Combination of the $\mathrm{K} \alpha$ and Bremsstrahlung spectroscopy will improve diagnostic accuracy of hot electron energy distribution function in the cone. In this study, Au was chosen as a tracer since it is one of representative

\footnotetext{
ae-mail: nishimu@ile.osaka-u.ac.jp
}

This is an Open Access article distributed under the terms of the Creative Commons Attribution License 2.0, which permits unrestricted use, distribution, and reproduction in any medium, provided the original work is properly cited. 


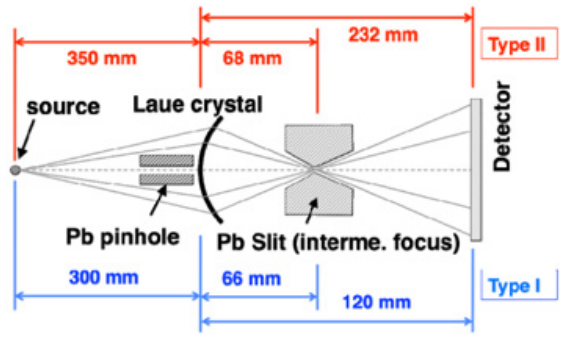

Figure 1. Schematic of the Laue spectrometer system and distances for Type-I and Type-II setups.

highest- $\mathrm{Z}$ materials available for the guide cone, thus better matching with $\mathrm{MeV}$-hot electrons than lower- $\mathrm{Z}$ tracers such as $\mathrm{Cu}$.

\section{HIGH ENERGY K $\alpha$ LINE SPECTROMETER}

A Laue spectrometer was developed to cover high energy range from Mo ( $\mathrm{K} \alpha_{1}: 17.48 \mathrm{keV}, \mathrm{K} \alpha_{2}$ : $17.37 \mathrm{keV})$ to $\mathrm{Au}\left(\mathrm{K} \alpha_{1}: 68.80 \mathrm{keV}, \mathrm{K} \alpha_{2}: 66.99 \mathrm{keV}\right) \mathrm{K} \alpha$ lines. As shown in Fig. 1 the spectrometer consists of a cylindrically curved Quartz (10-11) plate of $0.2 \mathrm{~mm}$ in thickness, $14 \mathrm{~mm}$ in height and $30 \mathrm{~mm}$ in width (direction corresponding to spectral dispersion). The detector can either be an imaging plate (IP) from Fujifilm [2] or a charge coupled device (CCD: Andor Model DH420-FO, 6.7 mm in height and $25 \mathrm{~mm}$ in width) with a fiber-optic plate coated with a CsI phosphor of $100 \mu \mathrm{m}$ thickness. The Quartz plate is bent with a radius of $170 \mathrm{~mm}$ in such that the diffracted X-rays are focused once at the intermediate slit. X-ray components propagating in a straightforward manner are prevented from irradiating the detector directly with a lead pinhole plate located in front of the crystal and a pair of lead shields located at the intermediate x-ray focus. To avoid influence of hard x-rays from plasma on the output signal, the whole body of the spectrometer and the detector are covered with lead shields. This Cauchois geometry effectively discriminates $0^{\text {th }}$-order component, stray X-rays and fluorescence from spectrometer components such as filters [3, 4]. By varying the distances from the crystal to the source and detector, this spectrometer can cover the energy range of either 10-60 keV (Type-I set) or 30-100 keV (Type-II set) (see Fig. 1 for details).

Absolute sensitivity of the Laue spectrometer was defined as follows. First, the overall sensitivity was calibrated using laser-produced-plasma (LPP) $\mathrm{K} \alpha$ lines and radioisotopes (RIs). For RIs, IP was used in place of the CsI/CCD because of the low-yield-rate of x-rays. Cross-calibration between the IPs and the CsI/CCD was performed at discrete energies. Second, to fully span the energy range, the spectral responses of the individual components of the spectrometer were theoretically calculated and compared to the experimental calibration data [5].

Absolute sensitivity for the Type-I setup was measured [6] at J-KAREN laser system at Japan Atomic Energy Agency, Kansai Photon Science Institute [7]. This system delivers a laser pulse of $800 \mathrm{~nm}$ wavelength, $1.8 \mathrm{~J}$ energy and $58 \mathrm{fs}$ duration. The pulse contrast ratio was typically $10^{-11}$. An $\mathrm{f} / 2.67$ gold-coated off-axis parabolic mirror was used to focus a $p$-polarized laser beam at an incident angle of $22.5 \mathrm{deg}$. relative to the target normal. 100- $\mu$ m-thick Mo and Ag planar targets were mounted on a motorized translation stage. The laser focal spot size was varied by translating the target along the laser beam to change laser irradiance. The absolute yield of $\mathrm{K} \alpha \mathrm{x}$-rays was measured with a backilluminated x-ray CCD operated in a single-photon counting mode. The spectral sensitivity of the CCD was absolutely calibrated separately by using radioisotopes.

The calibration for the Type-II was taken out with radioisotopes. The line emission at $59 \mathrm{keV}$ from ${ }^{241} \mathrm{Am}(370 \mathrm{kBq})$, and $77 \mathrm{keV}$ from ${ }^{226} \mathrm{Ra}(3.7 \mathrm{MBq})$ were used in this calibration. The calibration with ${ }^{226} \mathrm{Ra}$ was taken out at the OCTAVIAN facility at Osaka University. Due to a long 


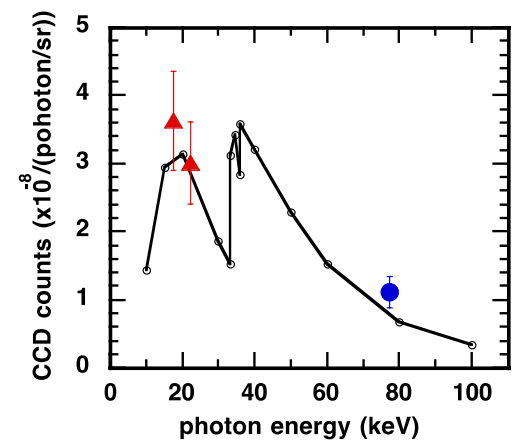

Figure 2. Overall sensitivity of Laue spectrometer system with CsI/CCD detector. The solid line represents the product of spectral sensitivities for the crystal and CsI/CCD. Experimental data points are also plotted for comparison.

exposure time, IP of Type BAS-TR2025 from Fujifilm was applied [8]. The typical exposure time was 20 to 140 hours. Due to the self-fading effect of IP, the spectral intensities recorded on IP are not in proportion to the exposure time. The absolute sensitivity and fading rate were calibrated in a time range from 5 min to 200 hrs by using the Fuji BAS 1800 IP scanner. The calibration was done at $59 \mathrm{keV}$ byusing ${ }^{241} \mathrm{Am}$; for other photon energy, the data in Ref. [9] is considered. In order to compare the data recorded with CsI/CCD in the experiment, a cross calibration between IP and CsI/CCD has also been made.

\section{COMPARISON OF SENSITIVITY WITH MODEL PREDICTIONS}

Spectral sensitivity for the $\mathrm{Au} \mathrm{K} \alpha$ line was obtained by comparing model prediction with the measurement. Diffraction efficiency for the Quartz crystal was derived with XOP code assuming the Takagi-Taupin model [10], and the spectral sensitivity for the CsI phosphor screen was referred from Ref. [11]. Figure 2 shows the comparison result. The solid line represents the product of calculated diffraction efficiency and spectral sensitivity of CsI. Absolute sensitivities calibrated with laser produced $\mathrm{K} \alpha$ lines and that for a radioisotope are respectively shown with triangles and a circle.

\section{K $\alpha$ LINE MEASUREMENT FOR AU-CONE-GUIDED TARGETS}

The absolute yield of $\mathrm{Au} \mathrm{K} \alpha$ line was measured in the fast ignition experimental campaign by using Gekko XII and LFEX lasers [12] at ILE, Osaka Univ. The target was a Au-cone CD shell. The thickness of the cone sidewall was $10 \mu \mathrm{m}$ and that of the tip was $6 \mu \mathrm{m}$. CD shell was typically $500 \mu \mathrm{m}$ in diameter and $7 \mu \mathrm{m}$ in thickness. The shell was driven with nine green-laser-pulses from GXII of $1.2 \mathrm{~ns}$ in duration and $2 \mathrm{~kJ}$ in total energy on target. LFEX laser irradiated the inner wall of the cone tip at the maximum compression of the shell. Energy and duration of the LFEX pulse were respectively $500+/-100 \mathrm{~J}$ and $1.5 \mathrm{ps}$, yielding intensity of $1.2 \times 10^{19} \mathrm{~W} / \mathrm{cm}^{2}$ at the cone tip. The density of the core is estimated to be around $10 \mathrm{~g} / \mathrm{cm}^{3}$ and density-radius product is around $0.02 \mathrm{~g} / \mathrm{cm}^{2}$.

Assuming a single Maxwellian electron velocity distribution and one-way travel of hot electrons through the cone, energy transfer efficiency (TE) of incident LFEX laser to hot electrons was derived according to Salzmann's model [13]. Figure 3 shows the TE given as a function of hot electron temperatures. A typical $\mathrm{Au} \mathrm{K} \alpha$ line spectrum observed with the LAUE spectrometer is inset there. Intense continuum line overlapping with the $\mathrm{K} \alpha$ line is due to Bremsstrahlung from hot electrons passing through the core plasma. Two plausible temperatures for the experimental conditions are shown for 


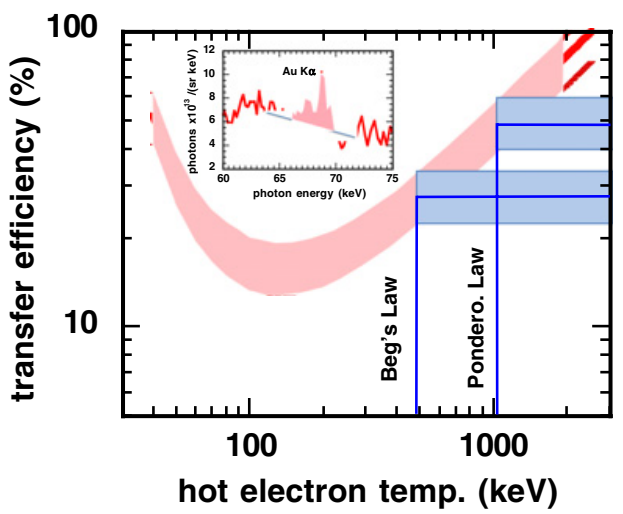

Figure 3. Energy transfer efficiency (TE) from LFEX laser to hot electrons as a function of hot electron temperature.

comparison. One is predicted by Beg's empirical law of $T_{\mathrm{h}}(\mathrm{keV})=215\left(I_{18} \lambda_{\mathrm{L}}^{2}\right)^{1 / 3}[14]$, and the other is by ponderomotive force law of $T_{\mathrm{h}}(\mathrm{keV})=512\left(\left(1+0.73 I_{18} \lambda_{\mathrm{L}}^{2}\right)^{1 / 2}-1\right)$ [15]. Here $I_{18}$ is the laser intensity in units of $10^{18} \mathrm{~W} / \mathrm{cm}^{2}$ and $\lambda_{\mathrm{L}}$ is the laser wavelength in micrometer. It is inferred that TE for the present conditions is around 30-50\%. This value can be compared to the case of $T_{\mathrm{h}}$ measured with an electron spectrometer set along the symmetrical axis of the cone in the forward direction of LFEX laser incidence. $T_{\mathrm{h}}$ predicted from the slope temperature at $10 \mathrm{MeV}$ electron kinetic energy was 4.1 MeV, resulting in TE over $100 \%$. Therefore we will attempt to measure the temperature of hot electrons directly at the interaction region by observing Bremsstrahlung continuum.

\section{SUMMARY}

$\mathrm{K} \alpha$ line spectroscopy, particularly for hard-X-ray region, has been proposed for quantitative measurement of cone-guided fast ignition targets. The $\mathrm{Au} \mathrm{K} \alpha$ line from the Au-cone guided target was observed and energy transfer efficiency was provided as a preliminary study. In near future, absolute measurement of hard $\mathrm{x}$-ray continuum will be made together with that of the K $\alpha$ line to improve accuracy of energy transfer measurements in the cone-guided fast ignition targets. Furthermore, detailed analysis with a hybrid modeling, namely combination of particle-in-cell (PIC) code and hydrodynamic code, will be made for $\mathrm{K} \alpha$ yield to improve understanding of hot electron generation, transport and deposition in the cone-guided target.

\section{References}

[1] C. D. Chen, P. K. Patel, D. S. Hey, A. J. Mackinnon, et al., Phys. Plasma 16, 082705 (2009)

[2] J. Miyahara, K. Takahashi, Y. Amemiya, et al., Nucl. Instrum. Methods 246, 572 (1986)

[3] Y. Cauchois, J. de Physique 3, 320 (1932)

[4] J. F. Seely, G. E. Holland, L. T. Hudson, et al., High Energy Density Physics 3, 263 (2007)

[5] Z, Zhang, H. Nishimura, T. Namimoto, et al., Rev. Sci. Instrum. 83, 053502 (2012)

[6] Z. Zhang, M. Nishikino, H. Nishimura, T. Kawachi et al., Optics Express 19, 4560 (2011)

[7] H. Kiriyama, M. Mori, Y. Nakai, T. Shimomura, H. Sasao, et al., Opt. Lett. 35, 1497 (2010)

[8] J. Miyahara, et al., Nuclear Instruments and Methods in Phys. Res. Sec. A 246, 572 (1986)

[9] A. L. Meadowcroft, C. D. Bentley, and E. N. Stott, Rev. Sci. Instrum. 79, 113102 (2008)

[10] XOP code: http://www.esrf.eu/computing/scientific/xop2.1/ 


\section{IFSA 2011}

[11] A W. Mengesha, T. D. Taulbee, R. D. Rooney, et al., IEEE Trans. Nuclear Sci. 45, 456 (1998)

[12] J. Kawanaka, et al., Journal of Physics: Conference Series 112, 032006 (2008)

[13] D. Salzmann, Ch. Reich, I. Uschmann, and E. Förster, Phys. Rev. E 65, 036402 (2002)

[14] F. N. Beg, A. R. Bell, A. E. Dangor, C. N. Danson, et al., Physics of Plasmas 4, 447 (1997)

[15] S. C. Wilks and W. L. Kruer, IEEE J. Quantum Electronics 33, 1954 (1997) 